\title{
Managed-Care-Ärztenetze: Rückblick auf das Jahr 2010 und Ausblick
}

\author{
Am 13. Managed-Care-Zukunfts-Workshop vom 9. bis 11. Dezember 2010 in Scuol \\ trafen sich verschiedene Vertreter von Managed-Care-Ärztenetzen, Vertreter des \\ Dachverbandes medswiss.net, der Hausärzte Schweiz und eines Versicherers zum \\ jährlichen Gedankenaustausch.
}

Zu Beginn des 13. Treffens in Scuol diskutierten die Teilnehmer die guten bzw. weniger guten Ereignisse des Jahres 2010. Hierbei prallten bereits die verschiedenen Ansichten aufeinander, wie Managed Care (MC) oder eben neu «Integrierte Versorgung» in $\mathrm{Zu}$ kunft aussehen soll. Je nachdem, welche Sicht vertreten wurde, war die Bilanz für das Jahr eher positiv oder negativ. Grundsätzlich waren aber alle erfreut, dass Managed Care verstärkt zum Thema wurde, sowohl in den Medien als auch in der Politik.

Als die Teilnehmer ihren Eindruck von der Tätigkeit des neuen Gesundheitsministers notieren sollten, wurde deutlich, dass sich viele noch keine Meinung bilden konnten. Es wurde jedoch festgehalten, dass grundsätzlich das Managed-Care-Prinzip auf Bundesebene grössere Unterstützung erhalten habe und sogar der Wille spürbar sei, MC zum Durchbruch zu verhelfen. Es sei aber konkret noch wenig hör- und spürbar; mit Ausnahme der Referendumsdrohungen verschiedener Interessenvertreter, vor allem von Ärzte-Kollegen, die MC als Untergang des freien Arztberufes sehen. Es sei aber für ein abschliessendes Urteil noch zu früh, nicht zuletzt wegen der heterogenen Interessen der Ärzteschaft, die es der Politik schwermachen, gesetzgeberisch rasch voranzukommen. Diese Grundstimmung kam dann besonders deutlich bei der Diskussion der anstehenden KVGRevision zum Ausdruck: Betont wurde, dass gesetzliche Regelungen, die den Markt behindern, grundsätzlich schlecht und nicht liberal seien. Zudem müssten

Die Arbeitsgruppe unterstützt die aktuell in den Eidgenössischen Räten diskutierte MC-Vorlage, weil darin schon lange geforderte, für die MCEntwicklung wesentliche Punkte enthalten sind:

- Verfeinerter, morbiditätsgerechter Risikoausgleich als Basis jeder Entwicklung des KVG;

- Der MC-Artikel basiert auf dem Vertragsprinzip zwischen Ärztenetzen und Krankenversicherern statt dem bisherigen Wahlprinzip; dank differenziertem Selbstbehalt, der so bemessen sein muss, dass die Grundversicherungsprämie nicht erhöht werden muss.

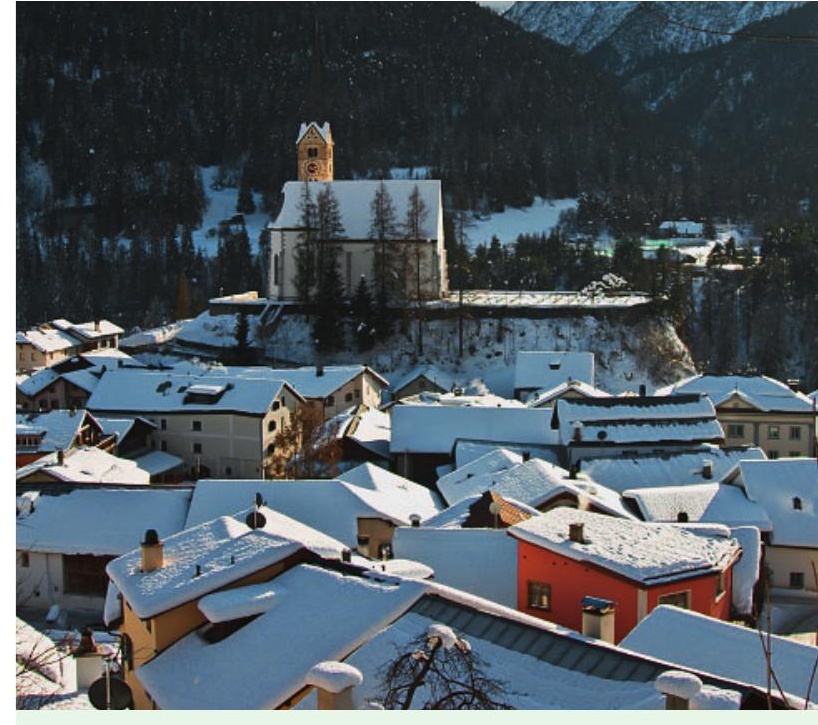

Im verschneiten Scuol fand zum 13. Mal ein ManagedCare-Zukunfts-Workshop statt.

aus politischem Kalkül und wegen der Befindlichkeit verschiedenen Ärztegruppierungen Begriffe wie Budgetmitverantwortung, MC-Obligatorium und Versicherer-Netze verständlicher diskutiert werden.

\section{Diskussion der KVG-Revisions-Vorschläge}

Der Präsident des Dachverbands med-swiss.net orientierte über die laufende Gesetzesrevision, über die Ausformulierung der einzelnen Artikel und was dies konkret bedeute. Er erläuterte auch, welche Punkte umstritten sind, teilweise drohen hier Organisationen mit dem Referendum. Das Wichtigste ist, das Hauptziel der Verankerung von MC im KVG nicht aus den Augen zu verlieren. Die Vertreterin von Hausärzte Schweiz zeigte eindrücklich auf, wie in verschiedenen Sitzungen zusammen mit Vertretern vom Forum Managed Care, bedeutenden Netzen und med-swiss.net versucht wurde, die interessierten Opinion Leaders der Krankenversicherer und der Politik $\mathrm{zu}$ informieren. Es besteht immer noch eine massive Verunsicherung und Unkenntnis über MC im allgemeinen und über die Inhalte der Managed-Care-

Vorlage im speziellen. 
Ausgiebig wurde über Eckwerte bzw. Killerkriterien diskutiert. Die zentrale Botschaft der MC-Gruppe Scuol ist: Die MC-Entwicklung darf nicht an einzelnen Kriterien scheitern. Es sollen Eckwerte formuliert werden und keine Killerkriterien, von denen man nicht mehr abweichen kann. Qualitätskriterien müssen vor Strukturkriterien gestellt werden. Da aber mehrheitlich davon ausgegangen wird, dass bei einem Referendum mit Volksabstimmung ein MC-Artikel abgelehnt werden könnte, müssen unverändert folgende Kernpunkte prioritär gefordert werden:

- Differenzierter Selbstbehalt von z.B. $10 \%$ bzw. $20 \%$ und 500 CHF bzw. 1000 CHF;

- Das Vertragsprinzip muss gelten;

- Neuer Risikoausgleich als Basis jeder Entwicklung.

Aufgrund dieser Überlegungen soll dem Risikoausgleich eventuell sogar mit einer separaten Gesetzesvorlage zum Durchbruch verholfen werden. Ausführlich diskutiert wurde, wie diese Botschaften bei der Bevölkerung und den Politikern publik gemacht werden können, denn der Mehrwert für MC-Versicherte ist immer noch nicht spürbar, ausser bei der Prämienreduktion.

\section{Grundsatzpapiere zur Information über das Wesen von Managed Care}

Es wurden in vier Arbeitsgruppen Grundsatzpapiere erarbeitet zu Fragen rund um die KVG-Revision, zur Integrierten Versorgung und zur MC-Information der Öffentlichkeit. Man war sich einig, dass die Botschaften ausser bei den eidgenössischen Politikern vor allem bei den netzeigenen Mitgliedern publik gemacht werden müssen. Es wurde festgehalten, dass bei der Ausarbeitung dieser Positionspapiere auf die internen Kritiker und MC-kritischen Spezialisten keine Rücksicht genommen werden soll.

Nach ausführlicher Diskussion und mehrfacher Redigierung konnten drei Papiere verabschiedet werden, die von allen Teilnehmern gutgeheissen wurden. Die Grundsatzpapiere sind auf der Homepage des Dachverbandes med-swiss.net (www.medswiss-net.ch) abrufbar. Es wurde festgehalten, wie die in den drei Papieren festgehaltenen Thesen zielgruppengerecht den Parlamentariern, allen Ärzten und der Bevölkerung via Publikationen zugänglich gemacht werden.

\section{Teilnehmer des Workshops}

Dres. med. Roman Buff (vmco, eastcare), Wolfgang

Czerwenka (argomed), Margot Enz Kuhn (argomed, Hausärzte Schweiz), Jörg Fritschi (LuMed, medswiss.net), Thomas Grüninger (Centramed), Felix Huber (mediX Zürich), Rainer Hurni (zmed), Urs Keller (PizolCare), Ueli Krebs (Igomed), André Müller (grisomed), Alfred Rösli (hawadoc), Andreas Schoepke (HARESO), Adrian Wirthner (mediX bern); Oliver Reich (Helsana), Lorenz Borer, Jörg Schläpfer und Rudolf Wartmann (Novartis Pharma Schweiz AG). Der Workshop wurde moderiert von Dr. H.J. Schlegel und unterstützt von der Firma Novartis.
Die vorgestellten Projekte und Präsentationen aus dem Jahre 2010

e-Health - Grundlage für integrierte Versorgung

Dr. med. Wolfgang Czerwenka, Wettingen, argomed ärzte

Gemeinsame Information der politischen Opinion Leaders im Jahre 2010 (msn-MFE-fmc)

Dr. med. Margot Enz Kuhn, Baden, Hausärzte Schweiz

KVG-Revision - was ist aus unseren Forderungen geworden?

Dr. med. Jörg Fritschi, Obernau, LuMed,

Präsident medswiss.net

Das Futuro Projekt - Medikamenteneinsatz: qualitativ und medizinisch optimiert

Dr. med. Felix Huber, Zürich, mediX-Zürich

Wieso auch gute Projekte gelegentlich scheitern können

Dr. med. Rainer Hurni, Zürich, Zmed

Die ASP-eKG Forschung in der Hausarztmedizin Dr. med. Urs Keller, Wangs, PizolCare

Bericht über die Info-Veranstaltung mit NR Ignazio Cassis Dr. André Müller, Küblis, Grisomed

Onlinepraxis

Dr. med. Adrian Wirthner, Bern, mediX-Bern

Koordination Kostenträger - Interinstitutionelles Case Management

Oliver Reich, Helsana

Medikamentenmanagement - Neurologie-QZ-ToolSchwindel

Ruedi Wartmann, Novartis Pharma Schweiz

\section{Projektpräsentationen}

Erneut wurden diverse Projekte von verschiedenen Netzen vorgestellt. Ein Schwerpunkt war e-Health und die diversen Umsetzungsprobleme, beginnend bei der elektronischen Krankengeschichte, über die elektronische Vernetzung, die Online-Sprechstunde bis zur Online-Terminierung und das Disease Management auf Basis einer elektronischen Plattform mit Hilfe speziell ausgebildeter und zertifizierter MPAs. Medikamentenmanagement und -sicherheit waren wie Forschung in der Hausarztmedizin weitere Diskussionspunkte.

\section{Fazit}

An den drei Tagen im zunehmend verschneiten Scuol wurde erneut weit weg vom Alltag eine produktive und offene Diskussion unter den führenden innovativen MC-Verantwortlichen der Deutschschweiz zusammen mit einem Krankenkassenvertreter geführt. Mit der zunehmenden Thematisierung des Begriffes Managed Care im Rahmen der KVG-Revision und dem offensichtlichen Erfolg des Scuoler-MCPositionspapiers 2008 kam dieses Jahr neue Dynamik in diese informelle Scuoler Gruppe. Es wurde versucht, gute Grundlagen zu schaffen, um Managed Care in der Schweiz auf allen Ebenen zum Durchbruch zu verhelfen. Zudem konnte erneut festgestellt werden, dass es in den verschiedenen Netzen sehr viele innovative Projekte gibt, an denen das eine oder andere Netz partizipieren kann. 